\title{
Retinitis pigmentosa: mutations in a receptor tyrosine kinase gene, MERTK
}

Retinitis pigmentosa (RP) is a heterogeneous genetic disorder of the eyes. RP is characterized by abnormalities of photoreceptors (rods and cones) or the retinal pigment epithelium (RPE) leading to progressive loss of vision. It starts with the night blindness or defective dark adaptation in patients followed by constriction of the peripheral visual fields. Eventually, late in the course of the disease, the patients lose their central vision. The diagnosis of RP is made when a patient has rod dysfuntion as measured by dark adaptation or electroretinogram (ERG) monitoring, progressive loss in photoreceptor function and loss of peripheral vision with bilateral involvement. In the early stage of the disease, the fundus (the bottom of eyes), appears normal. As the disease progresses, the fundus shows arteriolar narrowing, fine dust-like intraretinal pigmentation, and loss of pigment from the RPE. The retina in a RP patient at an advance stage of the disease is characterized by the presence of intraretinal and preretinal clumps of black melanin pigments appearing like bone spicules, markedly attenuated retinal vessels, loss of RPE and paleness of the optic nerve (Pagon 1993).

Electroretinography determines the functional status of the photoreceptors. Electroretinography is performed by using a double electrode contact lens placed on the cornea. After light stimulation of the retina, an electrical potential arises which represents a composite response of millions of retinal cells, and the electrical output is amplified and displayed on an oscilloscope. Rod function is reflected by 
responses obtained under dark-adapted conditions whereas cone function is reflected by responses observed under light-adapted conditions. The two types of responses can be separated from each other, and the type and the extent of rod and/or cone involvement in the disease can be assessed. RP patients in an advance stage of the disease have no detectable rod and cone responses whereas young RP patients show early and severe impairment of pure rod responses (Pagon 1993).

RP is classified into non-syndromic (simple) and syndromic categories. In the non-syndromic category, the disease manifests only in the eyes, whereas in the syndromic category, other organs are also affected. A good example of syndromic RP is Usher syndrome where patients in addition to RP have hearing impairment. Non-syndromic RP can be familial or sporadic. Non-syndromic RP can be inherited as an autosomal dominant (AD), autosomal recessive (AR), X-linked recessive (XLR) or rare digenic (RDG) traits. Syndromic RP can also be caused by mutations in the mitochondrial genome.

The population incidence of RP ranges from 1 in 3,500 to 1 in 4,000 in the western countries (Pagon 1993; Dryja and Li 1995). Sporadic RP (sRP) represents 40-50\% of all RP cases. RP is known to be present in all ethnic groups and races. Autosomal dominant RP (adRP), autosomal recessive RP (arRP) and X-linked recessive RP (XLRP) represent 15-25\%, 5-20\% and 5-15\% of all RP cases, respectively (Pagon 1993; Dryja and Li 1995). Linkage analysis and other mapping techniques have revealed 34 loci for all forms of RP. There are 12 known loci for adRP located on chromosome 1q13-q23 (RP18), 3q21-q24, 6p21·2-cen (RP7), 7p15-p13 (RP9), 7q31·3 (RP10), 8q11-q13 (RP1), 11q13, 14q11.2 (RP27), 17p13.3 (RP13), 17q22 (RP17), 19q13.3 and 19q13.4 (RP11). Of 12 known loci for adRP, genes for only six loci are known so far. Rhodopsin (RHO), peripherin (RDS), RP1 protein, retinal outer segment membrane protein 1 (ROM1), neural retina lucine zipper transcription factor (NRL) and cone-rod otx-like photoreceptor homeobox transcription factor (CRX or CORD2) are mutated in RP cases mapped to loci on chromosome 3q21-q24, 6p21·2-cen, 8q11-q13, 11q13, 14q11.2 and 19q13·3, respectively.

There are 16 known loci for arRP located on chromosome 1p31 (RP20), 1p21-p22 (RP19), 1q31q32·1 (RP12), 2p11-p15 (RP28), 2q31-q33 (RP26), 2q37·1, 3q21-q24, 4p16·3, 4p12-cen, 5q31·2-q34, 6 p21.3 (RP14), 6cen-q15 (RP25), 10q23, 15q22, 15q26, and 16p12·1-p12·3. Of these loci, genes for 11 loci have been cloned and characterized for mutations so far. Retinal pigment epithelium-specific $65 \mathrm{kDa}$ protein (RPE65), ATP-binding cassette transporter-retinal (ABCR), crumbs homologue 1 (CRB1), arrestin (SAG), rod cGMP phosphodiesterase $\beta$-subunit (PDE6B), rod cGMP gated channel $\alpha$-subunit (CNGA1), cGMP phosphodiesterase $\alpha$-subunit (PDE6A), tubby-like protein 1 (TULP1), RPE-retinal G protein-coupled receptor (RGR) and cellular retinaldehyde-binding protein (RLBP1) are mutated in RP cases mapped to loci on chromosome 1p31, 1p21-p22, 1q31-q32·1, 2q37·1, 4p16·3, 4p12-cen, 5q31·2-q34, 6p21·3, 10q23 and 15q26, respectively. Interestingly, mutations in rhodopsin gene cause adRP as well as arRP mapped to chromosome 3q21-q34. Further, mutations in RPE65 gene also cause Leber congenital amaurosis (LCA). LCA is an autosomal recessive retinal dystrophy which is characterized by total blindness or greatly impaired vision at birth or within few weeks of life.

Of six known loci for XLRP, genes for only two loci are known. Mutations in retinitis pigmentosa GTPase regulator (RPGR) and novel protein with similarity to cofactor C genes cause XLRP in cases mapped to chromosome Xp21.1 (RP3) and Xp11.3 (RP2), respectively. Genes responsible for RP cases mapped to chromosome Xp22.13-p22.11 (RP15), Xp21·3-p21.2 (RP6), Xq22 (RP23) and Xq26-q27 (RP24) are yet to be identified. Mutations in RPGR and RP2 genes are the most common causes of XLRP, representing 25-30\% and 10-15\% of the XLRP cases, respectively. Digenic RP is caused by mutations in the heterozygous condition in both peripherin and ROM1 genes (Dryja et al 1997).

As $40-50 \%$ of all RP cases are sporadic, finding the genetic cause for these cases is a difficult task because no linkage analysis is possible. Recently, Gal et al (2000) have used a different and ingenious approach to find mutations in sRP cases. It is known that the mutation in a receptor tyrosine kinase gene, Mertk, in the Royal College of Surgeons (RCS) rat (D'Cruz et al 2000) causes defective phagocytosis of photoreceptor outer segments by the retinal pigment epithelium and retinal degeneration (D'Cruz et al 2000; also see Gal et al 2000). The human orthologue of the rat Mertk gene, MERTK, is located on chromosome 2q14.1 (Weier et al 1999). Gal et al (2000) determined the genomic structure of the MERTK gene and examined each of its 19 coding exons for mutations including the splice sites in 328 DNA samples from individuals with various retinal dystrophies and detected three different mutations in three individuals with RP. Their finding is the first evidence that a defect in the RPE 
phagocytosis pathway could lead to human retinal disease. They found a homozygous 5-bp deletion in exon 15 (2070delAGGAC) in a 45-year-old man who is the product of a consanguineous marriage and had onset of night blindness and poor vision in early childhood and currently has only central vision. The second patient has a homozygous $\mathrm{A} \rightarrow \mathrm{G}$ transition in the intron 10 splice acceptor site (IVS10$2 \mathrm{~A} \rightarrow \mathrm{G})$ in a 34-year-old woman whose normal parents are not related. She had onset of night blindness and poor vision in early childhood and currently has only central vision. Only her father was heterozygous for the splice-site mutation, suggesting that she received two copies of the same mutation from her father and has paternal uniparental isodisomy for chromosome 2; this was confirmed by microsatellite analysis. As expected, microsatellite markers from other chromosomes showed biallelic inheritance in her. The third patient, who is a 21 -year-old woman and had poor vision as a child and night blindness at age 12 , has a heterozygous premature termination codon (R651X). Taken together, the work of Gal et al (2000) demonstrated a new arRP locus at 2q14.1 using a non-conventional method. It is possible that the third patient has a second mutation in MERTK, most likely in the intronic segments as they failed to find mutation by direct sequencing of exons 1-19. Conversely, it is possible that both homozygous as well heterozygous mutations in MERTK cause RP as has been observed in the rhodopsin gene. Further, mutations in the MERTK gene in RP patients and a mutation in the retinal dystrophic RCS rat demonstrate that MERTK is essential for the proper function of the retina, and a defect in the phagocytosis of the photoreceptor outer segments by the RPE results in retinal degeneration in a subset of RP patients.

\section{References}

D'Cruz P M, Yasumura D, Weir J, Mathes M T, Abderrachim H, LaVail M M and Vollrath D 2000 Mutation of the receptor tyrosine kinase gene Mertk in the retinal dystrophic RCS rat; Hum. Mol. Genet. 9 645-652

Dryja T P, Hahn L B, Kajiwaara K and Berson E L 1997 Dominant and digenic mutations in the peripherin/RDS and ROM1 genes in retinitis pigmentosa; Invest. Ophthalmol. Vis. Sci. 38 1972-1982

Dryja T P and Li T 1995 Molecular genetics of retinitis pigmentosa; Hum. Mol. Genet. 4 1739-1743

Gal A, Yun L, Thompson D A, Weir J, Orth U, Jacobson S G, Apfelstedt-Sylla E and Vollrath D 2000 Mutations in MERTK, the human orthologue of the RCS rat retinal dystrophy gene, cause retinitis pigmentosa; Nature Genet. 26 270-271

Pagon R A 1993 Syndromic retinal dystrophy; in Retinitis pigmentosa: Present knowledge and outlook (ed.) E Rinaldi (Napoli: Liviana Medicina) pp 151-166

Weier H U, Fung J and Lersch R A 1999 Assignment of protooncogene MERTK (a.k.a.c-mer) to human chromosome 2q14.1 by in situ hybridization; Cytogenet. Cell Genet. 84 91-92

ARUn KUMAR

Department of Molecular Reproduction, Development and Genetics, Indian Institute of Science, Bangalore 560 012, India

(Email, karun@hamsadvani.serc.iisc.ernet.in) 